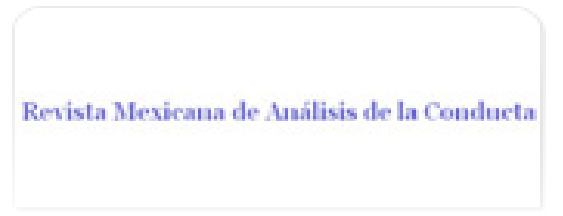

Revista Mexicana de Análisis de la Conducta ISSN: 0185-4534

editora@rmac-mx.org

Sociedad Mexicana de Análisis de la Conducta México

García-Leal, Óscar; Díaz Lemus, Carlos A.; Alfaro Hernández, Luís; Saldivar Olivares, Gamaliel Sensibilidad al riesgo en ratas (rattus norvegicus): efecto de la magnitud de reforzador y la probabilidad de reforzamiento

Revista Mexicana de Análisis de la Conducta, vol. 33, núm. 2, 2007, pp. 167-181

Sociedad Mexicana de Análisis de la Conducta

Guadalajara, México

Disponible en: http://www.redalyc.org/articulo.oa?id=59333204

- Cómo citar el artículo

- Número completo

- Más información del artículo

Página de la revista en redalyc.org

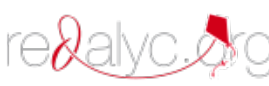

Sistema de Información Científica

Red de Revistas Científicas de América Latina, el Caribe, España y Portugal Proyecto académico sin fines de lucro, desarrollado bajo la iniciativa de acceso abierto 


\title{
SENSIBILIDAD AL RIESGO EN RATAS (RATTUS NORVEGICUS): EFECTO DE LA MAGNITUD DE REFORZADOR Y LA PROBABILIDAD DE REFORZAMIENTO
}

\author{
RISK-SENSITIVITY IN RATS (RATTUS NORVEGICUS): \\ EFFECT OF THE MAGNITUDE AND PROBABILITY \\ OF REINFORCEMENT
}

\author{
ÓSCAR GARCÍA-LEAL, CARLOS A. DÍAZ LEMUS, LUÍS ALFARO \\ HERNÁNDEZ Y GAMALIEL SALDIVAR OLIVARES \\ CENTRO DE ESTUDIOS E INVESTIGACIONES EN COMPORTAMIENTO \\ UNIVERSIDAD DE GUADALAJARA
}

\begin{abstract}
RESUMEN
En una tarea experimental de elección libre con ratas (Rattus norvegicus) se analizó el efecto de la magnitud de reforzador y la probabilidad de reforzamiento. Se manipuló en cada una de las alternativas de respuesta ambas variables, igualándose en ambas alternativas la cantidad de reforzador obtenido al final de la sesión. Inicialmente los animales fueron entrenados en dos posibles alternativas de respuesta de manera secuencial (elección forzada): 1) obtener con una alta probabilidad una cantidad de reforzador baja (bajo riesgo) y 2) obtener con una baja probabilidad una magnitud de reforzador alta (alto riesgo). Tras este entrenamiento, se presentó a los sujetos de manera simultánea ambas alternativas (elección libre). En general se observó preferencia por la alternativa etiquetada como bajo riesgo. Sin embargo, un análisis detallado revela un efecto de interacción entre magnitud de reforza-
\end{abstract}

1. Dirección de correspondencia: Óscar García Leal. Centro de Estudios e Investigaciones en Comportamiento, Universidad de Guadalajara. C/ Francisco de Quevedo \#180, Col. Arcos Vallarta. 44130, Guadalajara, Jalisco, México. Teléfono y Fax: (52 33) 3818 0736. Correo electrónico: oscarg|@cencar.udg.mx 
dor y probabilidad de reforzamiento que arroja luz acerca de la propensión al riesgo que las ratas mostraron en el experimento.

Palabras clave: sensibilidad al riesgo, magnitud de reforzador, probabilidad de reforzamiento, elección libre.

\begin{abstract}
Using a free-choice experimental task with rats (Rattus norvegicus), the effect of the magnitude and the probability of reinforcement was studied. Both variables were manipulated in two different alternatives of response, being equal the quantity of reinforcer on each alternative at the end of the experimental session. Initially, the rats were sequentially trained on two alternatives of response (forced-choice): 1) to get with high probability of reinforcement less magnitude of reinforcer (risk aversion) and 2) to get with low probability of reinforcement more quantity of reinforcer (risk prone). After the training, both alternatives of response were simultaneously presented (free-choice). In general, rats were risk averse. Nevertheless, a fine-grain analysis shows an interaction effect between both variables considered. This effect conveys about the possible risk propensity of the rats when these variables are combined into the same experimental preparation.
\end{abstract}

Keywords: risk sensitivity, magnitude of reinforcer, probability of reinforcement, free-choice.

La elección de una de dos posibles alternativas de respuesta depende, cuando menos, de dos factores fundamentales: la cantidad promedio de reforzador obtenido (Couvillon, Lee \& Bitterman, 1991) y la variabilidad en la disponibilidad del mismo (Bateson \& Kacelnik, 1995; Caraco, Martindale \& Whittman, 1980; Kacelnik \& Bateson, 1996; Fischer, Couvillon \& Bitterman, 1993; Real, 1981).

La sensibilidad al riesgo (Houston, 1991; Stephens, 1981), o la disposición de los animales por elegir una de entre varias posibles alternativas de respuesta más o menos arriesgadas, ha sido objeto de estudio de, cuando menos, psicólogos y ecólogos conductuales. En general, e independientemente de la disciplina desde la que se haya abordado su análisis, las situaciones experimentales de elección libre han sido frecuentemente utilizadas para el estudio de la sensibilidad al riesgo, tanto en animales inferiores como en humanos.

Sin embargo, el modo en el que se ha operativizado el riesgo asociado a cada una de las alternativas de respuesta y, en general, el modo en el que se ha abordado el estudio de la sensibilidad al riesgo, difiere sustancialmente en función de la disciplina desde la que se aborde su estudio. Para el caso que nos 
ocupa, consideraremos únicamente las diferencias existentes entre la aproximación psicológica y los estudios abordados por ecólogos conductuales.

La primera diferencia estriba en las especies utilizadas en los estudios. Los psicólogos han utilizado tradicionalmente humanos (Jacobs \& Hackenberg, 1996; Pietras, Locey \& Hackenberg, 2003), ratas (Kagel, MacDonald, Battalio, White \& Green, 1986) o pichones (Hamm \& Shettleworth, 1987), en tanto que desde la ecología conductual se ha estudiado fundamentalmente el comportamiento de animales inferiores, tales como abejas (Couvillon \& Bitterman, 1980; Shafir, Wiegmann, Smith \& Real, 1999; Shapiro, 2000), diferentes especies de aves (Caraco, 1981, 1982, 1983; Moore \& Simm, 1986) y peces (Roche, Dravet, Bolyard \& Rowland, 1998).

Además los estudios realizados difieren en los procedimientos experimentales utilizados. Los psicólogos habitualmente se han aproximado al estudio de la sensibilidad al riesgo utilizando procedimientos de operante libre hasta alcanzar un estado estable en las elecciones de los sujetos de estudio. Para ello, se presentan largas series de entrenamiento durante un elevado número de sesiones. Los estudios realizados desde la ecología conductual constan de un menor número de sesiones con una cantidad reducida de ensayos discretos.

Más interesante resulta la diferente manera en que desde ambas aproximaciones se ha evaluado el riesgo. Los psicólogos, siguiendo la tradición operante, han incorporado programas de reforzamiento que conducen a resultados constantes (bajo riesgo) o variables (propensión al riesgo); así por ejemplo, se han utilizado programas de intervalo fijo en una alternativa de respuesta, en tanto en la otra se utiliza un programa de intervalo variable con un promedio de duración del intervalo equivalente al utilizado en la otra alternativa de respuesta. En otros estudios (Case , Nichols y Fantino, 1995; García-Leal; Saldivar y Díaz, en prensa; Ha, Lehner y Farley, 1990; Zabludoff, Wecker y Caraco, 1988) se ha manipulado la demora de reforzamiento asociada a cada una de las alternativas de respuesta disponibles: demora constante para la alternativa asociada con bajo riesgo y demora variable para aquélla que recoge una mayor propensión al riesgo.

Ecólogos conductuales, por el contrario, han manipulado fundamentalmente la probabilidad de reforzamiento asociada a cada alternativa de respuesta y la magnitud de reforzador asociado. Caraco, Martindale \& Whittman (1980) encontraron que, dadas dos alternativas de respuesta disponibles, con la misma magnitud de reforzador obtenido en cada una de ellas tras una sesión experimental, animales de diferentes especies discriminan entre ambas alternativas de respuesta en función de la probabilidad de reforzamiento asociada a cada una de ellas (Caraco, 1981, 1983; Kacelnik \& Bateson, 1996).

Considerando las diferencias existentes entre ambas aproximaciones, parece razonable que los resultados obtenidos hayan resultado frecuentemente contradictorios. 
En general, la investigación realizada con ratas parece señalar que éstas muestran una baja propensión al riesgo, observándose un sesgo hacia la alternativa cuyo resultado se presenta de manera constante. Desde la ecología conductual se ha señalado repetidamente que la sensibilidad al riesgo de los animales está determinada, fundamentalmente, por su estado de necesidad de alimento o agua o, dicho de otro modo, por su estado de privación-saciedad (Stephens \& Krebs, 1986; Kacelnik \& Bateson, 1996). De este modo, los animales tenderán a mostrarse conservadores o poco propensos al riesgo cuando dispongan de nutrientes suficientes fuera de la situación experimental. Por el contrario, mostrarán una propensión al riesgo cuando la cantidad de agua o alimento obtenido durante la sesión experimental sea fundamental para garantizar sus posibilidades de supervivencia.

El presente estudio, utilizando ratas como sujetos experimentales, analizó el efecto que la probabilidad de reforzamiento y la magnitud de reforzador tienen sobre la sensibilidad al riesgo. Para ello, se utilizó un procedimiento experimental semejante al utilizado por ecólogos conductuales, pero en condiciones controladas de laboratorio. De este modo, el objetivo del presente estudio fue contrastar el efecto que sobre la preferencia de las ratas en la elección entre una de dos posibles alternativas de respuesta, ambas con igual valor esperado, tienen dos de los factores que desde la ecología conductual se han señalado relevantes en el estudio de la sensibilidad al riesgo. El estudio abunda, de este modo, en la comprensión desde una aproximación experimental llevada a cabo en condiciones controladas de laboratorio, de los factores señalados por estudios realizados por ecólogos conductuales en condiciones con, a priori, mayor valor ecológico.

En las diferentes sesiones experimentales, en una situación de elección libre, las ratas podían elegir entre una alternativa de respuesta con una probabilidad de reforzamiento baja, pero una magnitud de reforzador asociada alta, o una alternativa con una probabilidad de reforzamiento alta, pero una magnitud de reforzador asociada baja, igualándose la cantidad de reforzador obtenido al final de cada una de las sesiones experimentales en las dos alternativas de respuesta. En el primer caso, probabilidad baja y magnitud de reforzador alta, se habla de una conducta de riesgo, y en el segundo, probabilidad de reforzamiento alta y menor cantidad de reforzador, se habla de una conducta de noriesgo (Hamm \& Shettleworth, 1987; Weber, Shafir \& Blais, 2004).

\section{MÉTODO}

Sujetos

6 ratas hembra de la cepa wistar (Rattus norvegicus) experimentalmente ingenuas de 5 meses de edad en el momento de iniciarse el experimento, so- 
metidas a un régimen de privación de agua con 30 minutos de acceso diarios y acceso libre a comida durante el experimento.

\section{Aparatos}

Se utilizaron dos cajas operantes de experimentación marca MED-PC, modelo ENV-007, con un área de trabajo de $30 \mathrm{~cm}$ de largo $\times 25 \mathrm{~cm}$ de ancho x $21 \mathrm{~cm}$ de alto. En la pared frontal de la caja se localizaba una puerta de acceso de poliuretano transparente. La pared distal estaba compuesta del mismo material. El lateral derecho de la caja estaba conformado por tres vías de acero inoxidable en los que se insertaron los componentes utilizados en el estudio. En la vía central se insertó un dispensador de agua de tipo brazo modelo ENV-202M, a $2.5 \mathrm{~cm}$ del suelo, con capacidad para dispensar 0.04 cc de agua en cada administración. En ese mismo lateral, en cada una de las vías laterales se insertó una palanca retráctil, modelo ENV-112CM, a 7 $\mathrm{cm}$ del piso (rejilla). La presión que era necesario ejercer sobre cada una de las palancas para que se registrara una respuesta era de 0.2 newtons. Sobre cada una de las palancas, a $12 \mathrm{~cm}$ del piso, se instaló un foco luminoso, modelo ENV-221M, que se activaba cuando la palanca correspondiente estaba disponible.

\section{Procedimiento}

Se utilizó un procedimiento experimental divido en 3 fases. En la primera de las fases (moldeamiento) se entrenó a los animales a emitir una respuesta sobre una palanca para obtener el reforzador (0.04cc de agua). Se utilizó un programa de reforzamiento de razón fija 1 , con probabilidad de reforzamiento igual a 1, durante un total de 5 sesiones de 30 minutos de duración. Posteriormente, se inició el estudio experimental: fases 2 y 3 .

La fase 2, o fase de entrenamiento, estuvo constituida por un total de 14 sesiones de entrenamiento. En cada una de estas sesiones se entrenó a los animales en cada una de las alternativas de respuesta (riesgo y no-riesgo) de manera sucesiva durante un total de 20 ensayos en cada una de ellas. Veinte segundos después de iniciarse cada una de las sesiones experimentales se presentaba una de las palancas retráctiles y encima de ella se encendía el foco luminoso. Al emitir una respuesta el animal, la palanca se retraía, se apagaba el foco luminoso y se proporcionaba el reforzador con la magnitud y probabilidad correspondiente para cada uno de los grupos experimentales. El dispensador de agua ascendía y descendía de manera continua tantas veces como gotas de agua se hubieran de entregar en función de la condición experimental (véase Tabla 1). Veinte segundos después de haberse emitido la respuesta y retraído la palanca ésta aparecía de nuevo. Este ciclo se repitió durante un periodo de 20 exposiciones. Durante este entrenamiento la luz general de la caja permanecía siempre encendida. Finalizado el entrena- 
miento en una de las palancas se iniciaba un periodo de tiempo fuera de 2 minutos de duración durante el cual ambas palancas estaban retraídas y los focos luminosos y la luz general de la caja se mantenían apagados. Transcurrido este periodo se iniciaba el entrenamiento en la otra palanca.

Se conformaron 3 grupos: 2 experimentales (GE1 y GE2) y un grupo control (GC). Se controló tanto el color de la luz asociado a cada una de las posiciones en las que aparecían las palancas como la posición de la palanca inicialmente entrenada, en cada uno de los grupos. La luz que se presentaba encima de cada una de las palancas tenía como finalidad señalar que dicha palanca estaba disponible. Para cada uno de los grupos definidos, a cada uno de los sujetos que los conformaban, se presentaba un color de luz diferente (rojo y verde) asociado a cada una de las palancas utilizadas: al primer sujeto se presentaba una luz roja asociada a la palanca izquierda y una verde a la palanca derecha, en tanto que el otro sujeto recibía la combinación contraria. Véase tabla 1 para una descripción detallada de cada uno de los grupos utilizados.

\begin{tabular}{|c|c|c|c|}
\hline Grupo & Valores & Palanca 1 & Palanca 2 \\
\hline GC & $\mathrm{P}($ rf. $) / \mathrm{mg}$ (rf.) & $1 / 0.04 \mathrm{cc}$ & $1 / 0.04 \mathrm{cc}$ \\
\hline GE\#1 & $\mathrm{P}(\mathrm{rf}.) / \mathrm{mg}$ (rf.) & $.2 / 0.16 \mathrm{cc}$ & $.8 / 0.04 \mathrm{cc}$ \\
\hline GE\#2 & $P(r f.) / m g(r f)$. & $.4 / 0.12 \mathrm{cc}$ & $.6 / 0.08 \mathrm{cc}$ \\
\hline
\end{tabular}

Tabla 1. Probabilidad de reforzamiento y magnitud de reforzador asociada a cada una de las dos opciones de respuesta disponibles en cada uno de los grupos. $p(r f$.$) , probabilidad de reforzamiento; m g(r f$.$) , magnitud de refor-$ zador.

Como se observa en la Tabla 1, el valor de probabilidad de reforzamiento y magnitud de reforzador de cada una de las alternativas para cada uno de los grupos establecidos fue ajustado de tal manera que, siendo ambos valores diferentes para cada una de las alternativas de respuesta el valor esperado (el producto entre la probabilidad de reforzamiento y la magnitud de reforzador) fuera igual. De este modo, se igualó al final de la sesión la cantidad de reforzador recibida en cada una de las alternativas de respuesta. Durante las sesiones de entrenamiento, los sujetos recibieron en cada una de las alternativas de respuesta entrenada, la misma cantidad de reforzador, si bien éste se distribuyó de diferente manera a lo largo de la sesión.

La tercera fase, o fase de prueba, consistió en la presentación simultánea de ambas palancas durante un total de 40 ensayos. Las sesiones experimentales se realizaron en días consecutivos. Ambas palancas se presentaban si- 
multáneamente veinte segundos después de iniciarse la sesión experimental. Durante el tiempo de presentación de cada una de las palancas la luz asociada a cada una de ellas permanecía encendida, así como la luz general de la caja. Cuando el animal emitía una respuesta de palanqueo en cualquiera de ellas, ambas palancas se retraían, y se apagaban ambos focos luminosos. 20 segundos después ambas palancas y focos se activaban de nuevo.

Durante esta fase se registró tanto el número de elecciones realizadas de cada una de las palancas como el tiempo de respuesta del animal en cada una de las 40 elecciones que conformaban cada una de las sesiones experimentales.

Se realizaron dos diferentes sesiones de prueba. En la primera de ellas los animales no recibieron reforzador en ninguna de las elecciones realizadas, independientemente de las magnitudes o probabilidad de reforzamiento entrenadas previamente. En la segunda sesión se proporcionó siempre una entrega de agua $(0.04 \mathrm{cc}$ ) en cada una de las elecciones del animal, de nuevo independientemente de las magnitudes o probabilidad de reforzamiento entrenadas previamente.

La realización de dos pruebas diferenciadas (sin y con presencia de reforzador) tuvo como objetivo analizar si el cambio en la probabilidad de reforzamiento entre las fases de entrenamiento $(p>0)$ y prueba 1 del estudio $(p=0)$ tuvo un efecto sobre la preferencia de las ratas entre las alternativas de respuesta disponibles. En la prueba 2 el cambio en la probabilidad de reforzamiento fue en sentido inverso a la prueba 1 , de tal manera que toda elección fue reforzada con una probabilidad igual a $1(p=1)$.

Entre ambas sesiones de prueba se intercalaron dos sesiones de entrenamiento (fase 2) con el objetivo de eliminar los posibles efectos que la primera sesión de prueba pudiera presentar sobre la ejecución de los animales en la segunda.

\begin{tabular}{|c|c|c|c|c|c|}
\hline \multicolumn{2}{|c|}{ \# de sesiones } & $1 . .14$ & 15,16 & 17,18 & 19,20 \\
\hline & & & & & \\
\hline Fase & $\begin{array}{c}(1) \\
\text { Moldeamiento }\end{array}$ & $\begin{array}{c}(2) \\
\text { Entrenamiento }\end{array}$ & $\begin{array}{c}(3 A) \\
\text { Test sin reforzador }\end{array}$ & $\begin{array}{c}(2) \\
\text { Entrenamiento }\end{array}$ & $\begin{array}{c}(3 B) \\
\text { Test con reforzador }\end{array}$ \\
\hline
\end{tabular}

Tabla 2. Sesiones de entrenamiento y test

Si bien cada grupo estuvo conformado únicamente por 2 ratas, el número de elecciones que las ratas realizaban en cada una de las sesiones de prueba justifica la interpretación de los resultados en términos estadísticos, en tanto se dispone de 160 elecciones para cada uno de los grupos en cada una de las pruebas realizadas y se considera que hay una relación de independencia entre cada elección durante la fase de pruebas, de tal suerte que 
la elección anterior no tiene efecto alguno sobre la elección siguiente, y sí el entrenamiento al que se sometió a las ratas en la fase 2 del estudio.

\section{RESULTADOS}

Se comenta la distribución de respuestas observada en ambas condiciones de prueba: sin y con reforzador tras la serie de sesiones de entrenamiento en ambas palancas. Asimismo, se mencionan los resultados de analizar las latencias de respuesta para cada una de las alternativas durante las últimas sesiones de entrenamiento. Se presentan resultados grupales correspondientes a las 160 elecciones que realizaron cada uno de los grupos definidos en cada una de las dos condiciones de prueba aplicadas.

Prueba 1: Sin reforzador durante la fase de prueba

En la Figura 1 se presenta la distribución de respuestas en ambas palancas para cada uno de los grupos utilizados. El grupo control (GC) mostró un sesgo estadísticamente significativo $\left(X^{2}(1)=6.4 ; p<0.05\right)$ hacia la palanca 1 (izquierda), localizada en la vía más alejada de la entrada a la caja, independientemente de que en ambas palancas durante el entrenamiento obtuviera en todos los casos el reforzador en la misma cantidad ( $0.04 \mathrm{cc}$ de agua) y con una probabilidad igual a 1 .

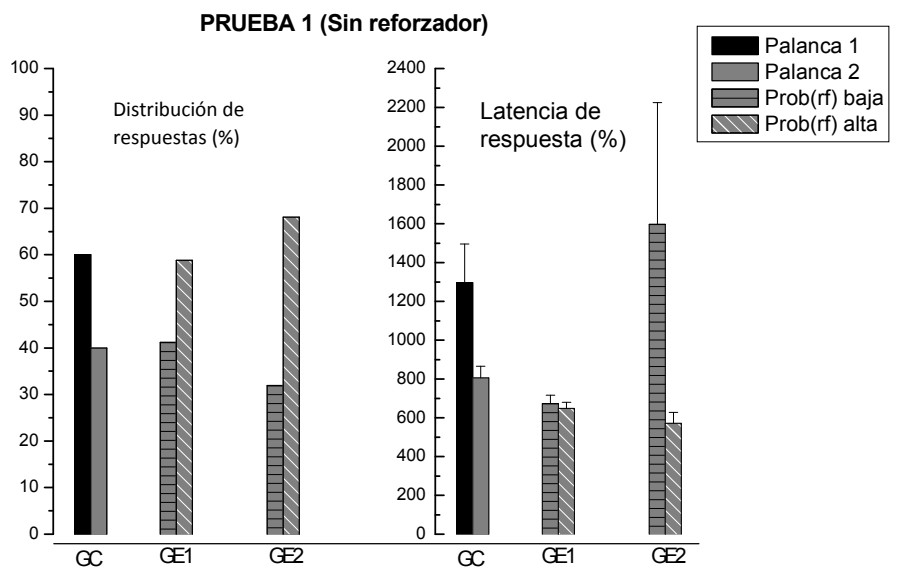

Figura 1. Porcentaje grupal promedio de distribución de respuestas en cada alternativa y latencia de respuesta promedio (en milisegundos) -se grafica el promedio de latencia observado y el error de estimación de la media- correspondiente a cada alternativa de respuesta en la Prueba 1 (sin reforzador). 
Ambos grupos experimentales mostraron una preferencia por la palanca con mayor probabilidad de reforzamiento pero menor magnitud, esto es, eligieron aquella alternativa de respuesta de menor riesgo. En ambos casos, difirió significativamente la proporción de respuestas emitidas sobre cada una de las alternativas disponibles, de la esperada si la elección hubiera sido realizada al azar (GE1: $\left.x^{2}(1)=4.9 ; p<0.05 ; G E 2: x^{2}(1)=21.025 ; p<0.01\right)$. Si la elección se hubiera realizado al azar cabría esperar igual número de respuestas en cada una de las dos alternativas disponibles. Las ratas discriminaron entre ambas alternativas de respuesta como resultado del entrenamiento previo en cada una de ellas.

La proporción de respuestas en cada una de las alternativas, para ambos grupos, también difirió cuando se comparó con la distribución esperable de acuerdo a la probabilidad de reforzamiento asociada a cada alternativa. En el caso del GE1, con probabilidad de reforzamiento 0.2 y 0.8 , la distribución observada difirió significativamente de la esperable en caso de que los animales ajustaran su comportamiento a la probabilidad asociada a cada alternativa, independientemente de la magnitud de reforzador obtenida $\left(X^{2}(1)=45.156\right.$; $p<0.01)$. El mismo resultado se observó para el GE2 $\left(X^{2}(1)=4.401 ; p<0.05\right)$.

No se observaron diferencias estadísticamente significativas en la latencia de respuesta asociada a cada una de las alternativas de elección en los grupos experimentales (GE1: $\mathrm{t}(158)=0.492 ; \mathrm{p}>0.05$; GE2: $\mathrm{t}(50.79)=1.627$; $\mathrm{p}>0.05$ ). Independientemente de la no existencia de diferencias estadísticamente significativas, para ambos grupos la latencia de respuesta correspondiente a la palanca que tenía asociada una menor probabilidad de reforzamiento (alternativa arriesgada) fue mayor. Esto se observó especialmente en el GE2, cuyas probabilidades asociadas a cada alternativa de respuesta diferían en menor medida que para el GE1. Sin embargo, la diferencia existente en el GE2 se debe a que se registraron elecciones con valores de latencia extremos que alteraron la representatividad del promedio. El valor promedio de latencia de respuesta para el G2 fue de 1597 msg., si bien el valor de la mediana fue de $610 \mathrm{msg}$. Véase Figura 1.

Sin embargo, sí se observaron diferencias en las latencias de respuesta para el grupo control. Este grupo, si bien mostró una preferencia hacia la palanca 1, presentó un tiempo de respuesta mayor para esta alternativa de respuesta $(\mathrm{t}(111.62)=2.361 ; \mathrm{p}=0.02)$. Una vez más, la representatividad del promedio de latencia correspondiente a la palanca 1 se ve alterada por latencias extremas en ensayos específicos. Es por esto que, si bien el valor promedio es de 1296 msg., el valor de la mediana es de únicamente $710 \mathrm{msg}$.

En general, se observaron latencias más altas para el grupo control que para ambos grupos experimentales, independientemente de los valores de probabilidad considerados. 
Prueba 2: Con reforzador durante la fase de prueba Durante la segunda serie de prueba utilizada en el estudio, se reforzó cada una de las elecciones del animal con $0.04 \mathrm{cc}$ de agua, independientemente de la probabilidad de reforzamiento y la magnitud de reforzador entrenada previamente en cada una de las alternativas de respuesta. Véase Figura 2.

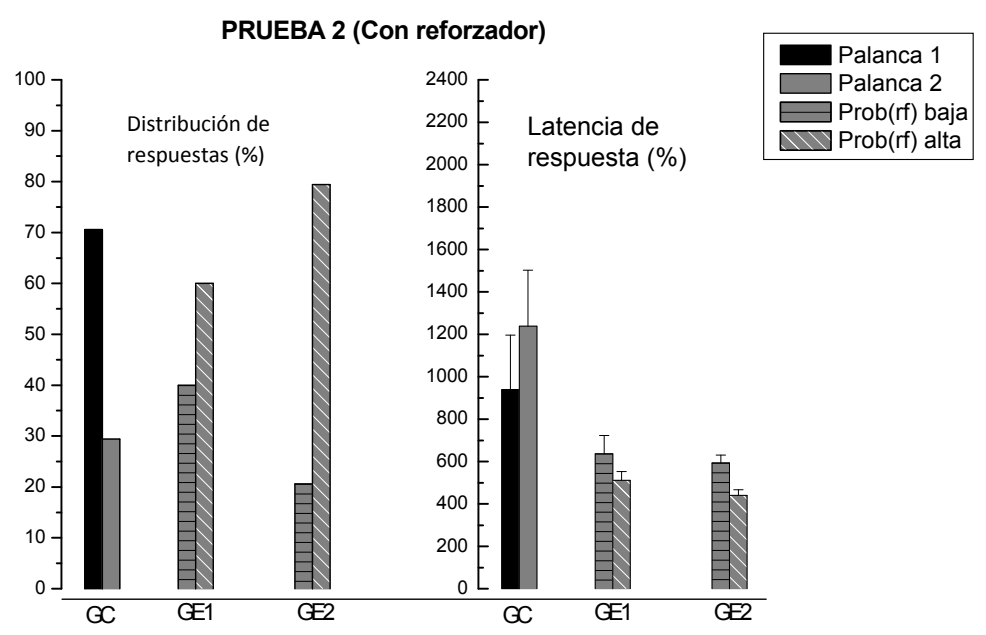

Figura 2. Porcentaje grupal promedio de distribución de respuestas en cada alternativa y latencia de respuesta (en milisegundos) -se grafica el promedio de latencia observado y el error de estimación de la media-correspondiente a cada alternativa de respuesta en la Prueba 2 (con reforzador).

De manera consistente con lo observado en la primera serie de prueba, durante la segunda serie los animales del grupo control (GC) también presentaron un sesgo estadísticamente significativo hacia la palanca situada en la vía más alejada de la entrada a la caja experimental $\left(X^{2}(1)=27.225 ; p<0.01\right)$.

Asimismo, los grupos experimentales mostraron también una preferencia por la alternativa que implicaba un menor riesgo $\left(G E 1: X^{2}(1)=6.4 ; p<0.05\right.$; GE2: $\left.X^{2}(1)=55.225 ; p<0.01\right)$ cuando la distribución de respuestas observada se comparaba con la distribución esperada si la elección se hubiera producido de manera azarosa. Esta diferencia también resultó significativa cuando la distribución de respuestas observada se comparaba con la esperable de acuerdo a las probabilidades de reforzamiento utilizadas en cada alternativa para ambos grupos (GE1: $\left.X^{2}(1)=40 ; p<0.01 ; G E 2: X^{2}(1)=25.026 ; p<0.01\right)$. 
De manera contraria a lo observado en la prueba 1, la latencia de respuesta correspondiente a cada una de las alternativas de respuesta no difirió significativamente para el grupo control $(t(158)=-0.688 ; p>0.05)$. Tampoco se observaron diferencias en el GE1 $(t(158)=1.441 ; p>0.05)$. Sin embargo, sí se observaron diferencias estadísticamente significativas en el GE2 $(t(50.79)=2.721 ; p<0.01)$. Véase Figura 2 .

Tal y como se observó en la prueba 1, en ambos grupos experimentales el promedio de latencia para la palanca con mayor probabilidad de reforzamiento fue menor que para la alternativa que tenía asociada menor probabilidad de reforzamiento. Una tendencia en sentido contrario se observó, sin embargo, para el grupo control.

De manera consistente a lo observado en la prueba 1 se observaron latencias más altas para el grupo control que para ambos grupos experimentales, independientemente de los valores de probabilidad considerados.

\section{DISCUSIÓN}

Se observaron resultados semejantes para ambas series de prueba utilizadas en el estudio. La presencia o ausencia del reforzador durante las pruebas no tuvo ningún efecto sobre la conducta de los animales, siendo por tanto el entrenamiento previo el determinante de la ejecución observada.

Los resultados obtenidos, de manera general y consistente con lo reportado en la literatura, parecen apoyar la hipótesis de que las ratas, ante dos alternativas de respuesta con diferente probabilidad y magnitud de reforzador asociada muestran preferencia por aquella alternativa de respuesta que implica asumir un menor riesgo (Kacelnik y Bateson, 1996). En una tarea de elección libre, tras ser entrenadas en dos alternativas de respuesta de manera independiente, las ratas seleccionaron con mayor frecuencia aquella alternativa que tuvo asociada durante el entrenamiento una mayor probabilidad de reforzamiento, independientemente de la magnitud de reforzador, en este caso cantidad de agua, que estuvo asociada a la elección. Como se ha mostrado, este resultado es independiente de que la elección durante la prueba estuviera seguida o no del reforzador.

EI GE1, como se puede observar en la Figura 3, presentó una distribución de respuestas en cada alternativa semejante en las dos series de prueba utilizadas (Prueba 1: 41.2\% - 51.8\%; Prueba 2: 40\% - 60\%).

Sin embargo, esta distribución uniforme no se observó en el GE2. En este grupo, la preferencia por la alternativa de respuesta que durante el entrenamiento tuvo asociada una mayor probabilidad de reforzamiento se incrementó significativamente cuando durante la prueba se presentó el reforzador asociado a la emisión de la respuesta $(79.4 \%)$, en relación a la preferencia 


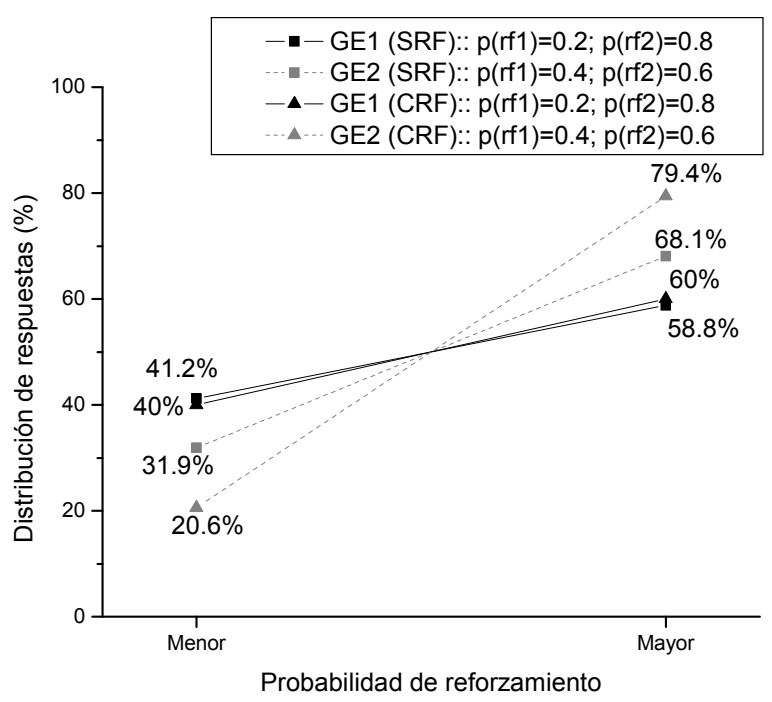

Figura 3. Distribución grupal promedio de respuestas entre ambas alternativas para ambas series de test utilizadas. Se observa un efecto de interacción entre la magnitud de reforzador y la probabilidad de reforzamiento para el grupo experimental.

por esta alternativa observada en la serie de prueba en la que las respuestas no fueron reforzadas (68.1\%). De manera complementaria, el número de elecciones de la alternativa con menor probabilidad de reforzamiento disminuyó $(20.6 \%)$ en relación a la serie de entrenamiento sin reforzamiento $(31.9 \%)$, independientemente de que esta alternativa tuvo asociada mayor cantidad de reforzamiento durante el entrenamiento.

Así pues, las ratas mostraron en ambos grupos experimentales una preferencia por la alternativa de menor riesgo. Sin embargo, como se observa en la Figura 3 esta preferencia fue más clara para el GE2 que para el GE1, aún cuando la diferencia entre las probabilidades asociadas a cada alternativa era menor. Este resultado muestra claramente un efecto de interacción entre magnitud de reforzador y probabilidad de reforzamiento. Este efecto de interacción se observa claramente cuando no se proporciona reforzador durante la fase de prueba y se intensifica cuando la emisión de una respuesta lleva asociada la obtención del reforzador.

La magnitud de reforzador que se obtiene en una alternativa de respuesta en cada elección reforzada es un factor determinante de la frecuencia de 
elección de esa alternativa, independientemente de la probabilidad de reforzamiento asociada. Si bien en general los resultados señalan que las ratas son poco arriesgadas en las elecciones realizadas, el porcentaje de elecciones de la alternativa con menor probabilidad de reforzamiento en el GE1 $(p(r f)=0.2)$ es cercano al $40 \%$ en ambas series de prueba. Durante la fase de entrenamiento las ratas obtuvieron $0.16 \mathrm{cc}$ en cada elección reforzada de esta alternativa de respuesta, en tanto obtuvieron $0.04 \mathrm{cc}$ en cada elección reforzada de la alternativa que tenía asociada una mayor probabilidad de reforzamiento.

Si comparamos el porcentaje de elecciones de la alternativa con menor probabilidad de reforzamiento en los GE1 y GE2 se observa que éste es superior para la alternativa con menor probabilidad de reforzamiento del GE1 $(p(r f)=0.2)$, que para la correspondiente del GE2 $(p(r f)=0.4)$, siendo de $41.2 \%$ vs $31.9 \%$ para la serie de prueba sin reforzador y de $40 \%$ vs $20.6 \%$ para la serie reforzada.

A la luz de estos datos parece conveniente concluir que las ratas lejos de presentar aversión al riesgo, muestran preferencia por la alternativa arriesgada cuando la magnitud de reforzador que es posible obtener en la alternativa arriesgada es mayor y difiere significativamente de la que es posible obtener en caso de elegir la alternativa de respuesta que presenta una mayor probabilidad de reforzamiento.

Este resultado parece contradictorio con la hipótesis que señala que los animales que requieren del reforzador para su subsistencia muestran mayor propensión al riesgo que aquellos que no requieren necesariamente de éste. En el estudio realizado, si bien las ratas estuvieron privadas de agua, una vez finalizada la sesión experimental tenían acceso libre al mismo por un periodo de tiempo suficiente para satisfacer los requerimientos biológicos. Aún así, mostraron una tendencia importante hacia la alternativa de respuesta que se consideró como más arriesgada. El resultado obtenido es contrario al observado en otros estudios en los que se concluye que las ratas muestran preferencia por la alternativa que implica mayor probabilidad de obtención del reforzador, denominada en este estudio 'bajo riesgo' (Kagel et al., 1986; Weber, Shafir \& Blais, 2004).

Por otra parte, en el estudio se observaron latencias ligeramente mayores asociadas a las alternativas de respuesta consideradas de 'alto riesgo'. Asimismo, se observó que el grupo control mostraba tiempos de respuesta sustancialmente mayores que los grupos experimentales. Si bien las diferencias observadas no fueron estadísticamente significativas, estos datos, cuando menos, contribuyen a señalar que las ratas fueron sensibles a las diferencias en probabilidad y magnitud de reforzamiento existentes entre las alternativas de respuesta disponibles, avalando el procedimiento experimental para el estudios de la sensibilidad al riesgo en ratas. 


\section{REFERENCIAS}

Bateson, M. y Kacelnik, A. (1995). Preferentes for fixed and variable food sources: variability in amount and delay. Journal of Experimental Analysis of Behavior, 63, 313-329.

Caraco, T. (1981). Energy budgets, risk and foraging preferences in dark-eyed juncos (Junco hyemalis). Behavioral Ecology and Sociobiology, 8, 213-217.

(1982). Aspects of risk aversion in foraging white-crowned sparrows. Animal Behaviour, 30, 719-727.

(1983). White-crowned sparrows (Zonotrichia leucophrys): Foraging preferences in a risk environment. Behavioral Ecology and Sociobiology, 12, 63-69.

Caraco, T.; Martindale, S. y Whittman, T.S. (1980). An empirical demonstration of risk sensitive foraging preferences. Animal Behaviour, 28, 820-830.

Case, D.A.; Nichols, P. y Fantino, E. (1995). Pigeon's preference for variable-interval water reinforcement under varied water budgets. Journal of Experimental Analysis of Behavior, 64, 299-311.

Couvillon, P.A. y Bitterman, M.E. (1980). Analysis of choice in honeybees. Animal Learning and Behavior, 28, 820-830.

Couvillon, P.A.; Lee, Y. y Bitterman, M.E. (1991). Learning in honeybees as a function of amount of reward: Rejection of the equal-asymptote assumption. Animal Learning \& Behavior, 19, 381-387.

Fischer, M.E.; Couvillon, P.A. y Bitterman, M.E. (1993). Choice in honeybees as a function of the probability of reward. Animal Learning \& Behavior, 21, 187-195.

García-Leal, O.; Saldivar, G. y Díaz, C.A. (en prensa). Efecto de la disponibilidad de recursos energéticos en la sensibilidad al riesgo en ratas (Rattus norvegicus). Acta comportamentalia.

Ha, J.C.; Lehner, P.N. y Farley, S.D. (1990). Risk-prone foraging behavior in captive grey jays Perisoreus canadensis. Animal Behavior, 39, 91-96.

Hamm, S.L. y Shettleworth, S.J. (1987). Risk aversion in pigeons. Journal of Experimental Psychology: Animal Behavior Processes, 13(4), 376-383.

Houston, A.I. (1991). Risk-sensitive foraging theory and operant psychology. Journal of Experimental Analysis of Behavior, 56, 585-589.

Jacobs, E.A. y Hackenberg, T.D. (1996). Humans' Choices in Situations of Time-Based Diminishing Returns: Effects of Fixed-Interval Duration and Progressive-Interval Step Size. Journal of the Experimental Analysis of Behavior, 65, 5-19.

Kacelnik, A. y Bateson, M. (1996). Risk-theories. Te effect of variante on foraging decisions. American Zoologist, 36, 402-434.

Kagel, J.H.; MacDonald, D.N.; Battalio, R.C.; White, S. y Green, L. (1986). Risk Aversion in Rats (Rattus norvegicus) Under Varying Levels of Resource Availability. Journal of Comparative Psychology, 100(2), 95-100.

Moore, F.R. y Simm, P.A. (1986). Risk-sensitive foraging by a migratory bird (Dendroica coronata). Experientia, 42, 1054-1056.

Pietras, C.J.; Locey, M.L. y Hackenberg, T.D. (2003). Human Risky Choice Under Temporal Constraints: Tests of an Energy-Budget Model. Journal of the Experimental Analysis of Behavior, 80, 59-75. 
Real, L.A. (1981). Uncertainty and pollinator-plant interactions: the foraging behavior of bees and wasps on artificial flowers. Ecology, 62, 20-26.

Roche, J.P.; Dravet, S.M.; Bolyard, K. y Rowland, W. (1998). Risk Sensitivity in Foraging Jack Dampsey Cichlids (Cichlasoma octofasciatum). Ethology, 104, 593602.

Shafir, S; Wiegmann, D.; Smith, B.H. y Real, L.A. (1999). Risk-sensitive foraging: Choice behavior of honey bees in response to variability in volume of reward. Animal Behaviour, 57, 1055-1061.

Shapiro, M.S. (2000). Quantitative analysis of risk sensitivity in honeybees (Apis mellifera) with variability in concentration and amount of reward. Journal of Experimental Psychology: Animal Behavior Processes, 26(2), 196-205.

Stephens, D.W. (1981). The logic of risk-sensitive foraging preferences. Animal Behaviour, 29, 628-629.

Stephens, D.W. y Krebs, J.R. (1986). Foraging theory. Princeton: Princeton University Press.

Weber, E.U.; Shafir, S. y Blais, A. (2004). Predicting risk sensitivity in humans and low animals: risk as variance or coefficient of variation. Psychological Review, 111(2), 430-445.

Zabludoff, S.D.; Wecker, J. y Caraco, T. (1988). Foraging choice in laboratory rats: constant vs variable delays. Behavioral Processes, 16, 95-110. 